\title{
Efecto de la modificación superficial de alambres delgados de acero inoxidable AISI 302 mediante plasma electrolítico sobre sus propiedades mecánicas ${ }^{(\cdot)}$
}

\author{
A. Gallegos*, C. Camurri*, C. Carrasco* y F. Soldera**
}

Resumen

En este trabajo se realizaron ensayos de aplicación de plasma electrolítico (PE) sobre alambres delgados de acero inoxidable AISI 302 en una solución inerte. Las probetas se sometieron a ensayos de tracción, con el fin de medir cambios en su resistencia mecánica; adicionalmente fueron evaluadas en sus cambios morfológicos y microestructurales. Se encontró que después de $10 \mathrm{~s}$ de aplicación de PE, la superficie de las probetas estaba uniformemente cubierta por nódulos y cráteres propios del ciclo de fusión y enfriamiento del PE; se evidenció un significativo afinamiento del grano superficial, llegando a ordenamientos cristalinos de tamaño menor a $200 \mathrm{~nm}$ y también se observó que la capa superficial afectada presentó un incremento de la resistencia a la tracción de al menos un 57 \% respecto al acero base.

Palabras clave

Plasma electrolítico; Electrólisis de alto voltaje; Nanoestructuración superficial; Nanogranos; Resistencia a la ruptura; Oxidación por plasma electrolítico.

\section{Effects of surface modification of thin wires of AISI 302 stainless steel by electrolytic plasma on its mechanical properties}

\begin{abstract}
In this work different tests using electrolytic plasma (EP) on thin wires of stainless steel AISI 302 in an inert solution were performed. Tensile tests were carried out in order to measure changes in the mechanical strength of the samples; moreover, both the morphological and microstructural changes also were evaluated. It was found that after $10 \mathrm{~s}$ of the application of EP, the samples surface was uniformly covered by nodules-like and craters similar to those found in the melting and cooling periods of EP. The results show a significant surface grain refinement, leading to crystalline arrangements with sizes less than $200 \mathrm{~nm}$ and also an increase in the samples tensile strength of at least $57 \%$ respect to steel base.
\end{abstract}

Keywords

Electrolytic plasma technology (EPT); Contact glow discharge electrolysis (CGDE); Surface nanostructuration; Nanograins; Rupture strength; Plasma electrolytic oxidation (PEO).

\section{INTRODUCCIÓN}

Es sabido que la presencia de granos pequeños favorece la resistencia mecánica de los materiales metálicos, fenómeno que se explica a partir de la clásica ecuación de Hall-Petch. Las potencialidades que resultan de esta relación han derivado en que muchas investigaciones se han dirigido a la tarea de afinar el grano en materiales metálicos, de una forma masiva, a través de técnicas que utilizan deformación plástica severa $^{[1]}$, por vía electroquímica ${ }^{[2]}$ o mediante transformaciones de fase por deformación ${ }^{[3 \text { y }}{ }^{4]}$. Otras investigaciones se han abocado a afinar el grano superficialmente, deformando mediante arenado ${ }^{[5-8]}$, bombardeo con esferas ${ }^{[9}$ y 10] , pulsos de ultrasonido ${ }^{[11]}$, atrición ${ }^{[12]}$ y más recientemente, mediante el uso de

(•) Trabajo recibido el día 26 de septiembre de 2011 y aceptado en su forma final el día 25 de junio de 2012.

* Departamento de Ingeniería de Materiales (DIMAT), Facultad de Ingeniería, Universidad de Concepción, C/ Edmundo Larenas 270 Casilla 160-C, Concepción, Chile.

** Departamento de Ciencia de Materiales, Universidad de Saarland, Saarbrücken. Alemania. 
deformación por jet de agua a alta velocidad ${ }^{[13]}$. También se ha logrado afinar el grano mediante la inclusión de aleantes ${ }^{[14]}$, o a través de ciclos rápidos de fusión local y enfriamiento, mediante la aplicación de pulsos láser ${ }^{[15]} \mathrm{o}$ en recubrimientos formados por plasma electrolítico ${ }^{[16]}$, siendo esta última técnica de nuestro interés y la utilizada en este trabajo. Ella considera la aplicación de elevados potenciales eléctricos continuos (VDC) sobre una celda de electrólisis, lo que da como resultado la formación de plasma sobre las burbujas de $\mathrm{O}_{2} \mathrm{OH}_{2}$, dependiendo de si el electrodo objetivo es el ánodo o cátodo, respectivamente. Para explicarlo, se considera la respuesta de la celda al aumentar continuamente el potencial aplicado, lo que resulta en una curva del tipo mostrado en la figura 1.

En ella se puede apreciar que a medida que se eleva el potencial aplicado a la celda, ésta pasa por 4 etapas que se describen a continuación: (i) Etapa 1: electrólisis convencional; en ambos electrodos se desprenden burbujas de $\mathrm{O}_{2}$ y/o $\mathrm{H}_{2}$, asociadas a las reacciones parciales de oxidación y reducción del agua, respectivamente. En esta etapa, la respuesta de la celda es lineal ${ }^{[17}$ y 18$]$, asimilable a una resistencia simple, constante. La superficie del electrodo de interés se calienta significativamente, al igual que el electrolito. (ii) Etapa 2: el electrodo objetivo se calienta tanto que el electrolito circundante ebulle, perdiéndose contacto y conductividad, aumentando en forma significativa la impedancia ${ }^{[19}$ y 20$]$; este fenómeno se agudiza mientras aumenta el voltaje aplicado, hasta llegar al rompimiento del dieléctrico y la generación de plasma. Es necesario hacer notar que la fenomenología en el contra-electrodo y en el electrolito no cambia, es decir, el aumento de impedancia de la celda es resultado del cambio de fenomenología en la superficie del electrodo de trabajo. (iii) Etapa 3: la pérdida de carga en la superficie del electrodo de trabajo es tal que se genera plasma. En este caso, las burbujas se encienden y se desprenden inmediatamente, debido a que se expanden bruscamente; se genera ruido en la señal eléctrica ${ }^{[21]}$. Al continuar aumentando el voltaje, el plasma se generaliza en la superficie, estableciéndose el arco eléctrico. (iv) Etapa 4: cuando se establece el arco eléctrico la impedancia vuelve a disminuir y cambian en forma significativa las frecuencias de radiación luminosa ${ }^{[22]}$.

Durante el PE (Etapa 3), los tiempos de descarga son del orden de $10^{-6} \mathrm{~s}$, en los cuales se alcanzan temperaturas del orden de $6 \times 10^{3} \mathrm{~K}$, fundiéndose el electrodo objetivo en el punto de contacto con la burbuja ${ }^{[16]}$. Cuando la burbuja se desprende del electrodo, la zona fundida queda expuesta a una mezcla de electrolito y vapor que se encuentra a temperaturas cercanas a $100^{\circ} \mathrm{C}$, lo que provoca su brusco enfriamiento, condición que genera ordenamientos cristalinos de corto alcance.

Esta técnica ha sido utilizada para la formación de recubrimientos catódicos ${ }^{[23 \text { y } 24] \text {, anódicos }}{ }^{[25]}$ y para síntesis en medio electrolito ${ }^{[26]}$, así como para usos ambientales ${ }^{[27]}$. Los recubrimientos formados poseen estructura amorfa y/o nanométrica y existen varios estudios que dan cuenta de sus propiedades. Sin embargo, no se han encontrado estudios que den cuenta de las propiedades mecánicas de la capa afec-

\section{Respuesta de celda electrolítica a altos voltajes}

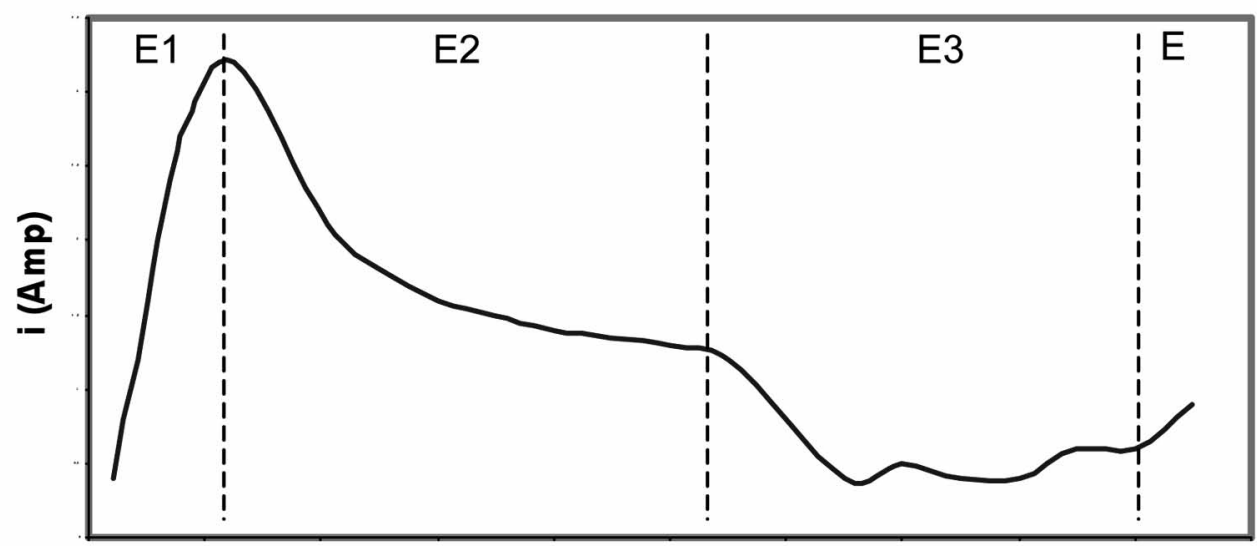

Potencial aplicado (Volts DC)

Figura 1. Curva típica de descargas de alto voltaje sobre electrolito.

Figure 1. Typical curve of high voltage discharges on electrolyte. 
tada por el PE, sin recubrimiento, que también debiese reordenarse nanoscópicamente.

En este trabajo se aplicó la técnica de PE sobre alambres delgados de acero inoxidable que fueron posteriormente sometidos a ensayos de tracción. Se eligió acero inoxidable por su elevado punto de fusión, relativa baja conductividad térmica respecto de un acero al carbono y composición que favorece la formación y estabilización de una capa nanoestructurada. Esto último por poseer cromo, que actúa como agente nucleante y níquel que impide el crecimiento de los granos.

\section{MÉTODO EXPERIMENTAL}

Para la realización de este trabajo se utilizó alambre comercial de acero inoxidable 302, de diámetro 0,5 mm y composición mostrada en la tabla I.

Tabla I. Composición de las probetas

Table I. Probes composition

\begin{tabular}{ccccccc}
\hline $\mathbf{C}$ & $\mathbf{S i}$ & $\mathbf{M n}$ & $\mathbf{P}$ & $\mathbf{S}$ & $\mathbf{C r}$ & $\mathbf{N i}$ \\
\hline$<0,080$ & $<0,75$ & 1,8 & 0,3 & $<0,03$ & 18,32 & 8,95
\end{tabular}

Este material fue montado como cátodo en una celda de electrólisis de $350 \mathrm{cc}$ de volumen efectivo. La celda estaba equipada con una fuente de poder de 2 KVA desarrollada en los laboratorios de la universidad.

Se efectuaron ensayos preliminares con el fin de evaluar las condiciones óptimas para la realización de los ensayos, observándose que al utilizar temperaturas de electrolito cercanas a ebullición $\left(95^{\circ} \mathrm{C}\right)$ y conductividades relativamente altas $(4 \mathrm{mS} / \mathrm{cm})$, se favorece la formación de plasma, estabilizándose a 230 VDC. Estas condiciones se definieron como las óptimas para la realización de los ensayos; por otro lado, mediante microscopía se observó que la zona afectada por el PE se homogeneiza después de $6 \mathrm{~s}$ de tratamiento, lo que permitió definir un tiempo de las experiencias de $10 \mathrm{~s}$.

Las probetas fueron sometidas a ensayos de tracción en un equipo Instron 4467, cuya sensibilidad es $1 \mathrm{~g}$, con velocidades de cabezal de 1 y $0,1 \mathrm{~mm} / \mathrm{min}$.

Se ensayaron muestras comerciales (muestras A) y con plasma (muestras B), registrándose como parámetro de control las respectivas cargas máximas. Para cada condición se realizaron tres ensayos de tracción.

Para caracterizar morfológicamente las probetas se utilizó microscopía electrónica de barrido. Se realizaron observaciones de las superficies antes y después de la aplicación de PE, luego de los ensayos de tracción y de las copas de fractura.

La caracterización estructural se realizó mediante metalografías de las muestras A y B. Posteriormente, mediante la técnica de erosión iónica (FIB) se obtuvo una muestra de la sección superficial, que fue analizada por microscopía electrónica con modo de barrido y transmisión (STEM).

\section{RESULTADOS}

\subsection{Morfología superficial}

En el caso de las muestras comerciales (A) y de las tratadas con PE (B), las superficies fueron observadas mediante microscopía electrónica de barrido (MEB), obteniéndose las morfologías mostradas en la figura 2.
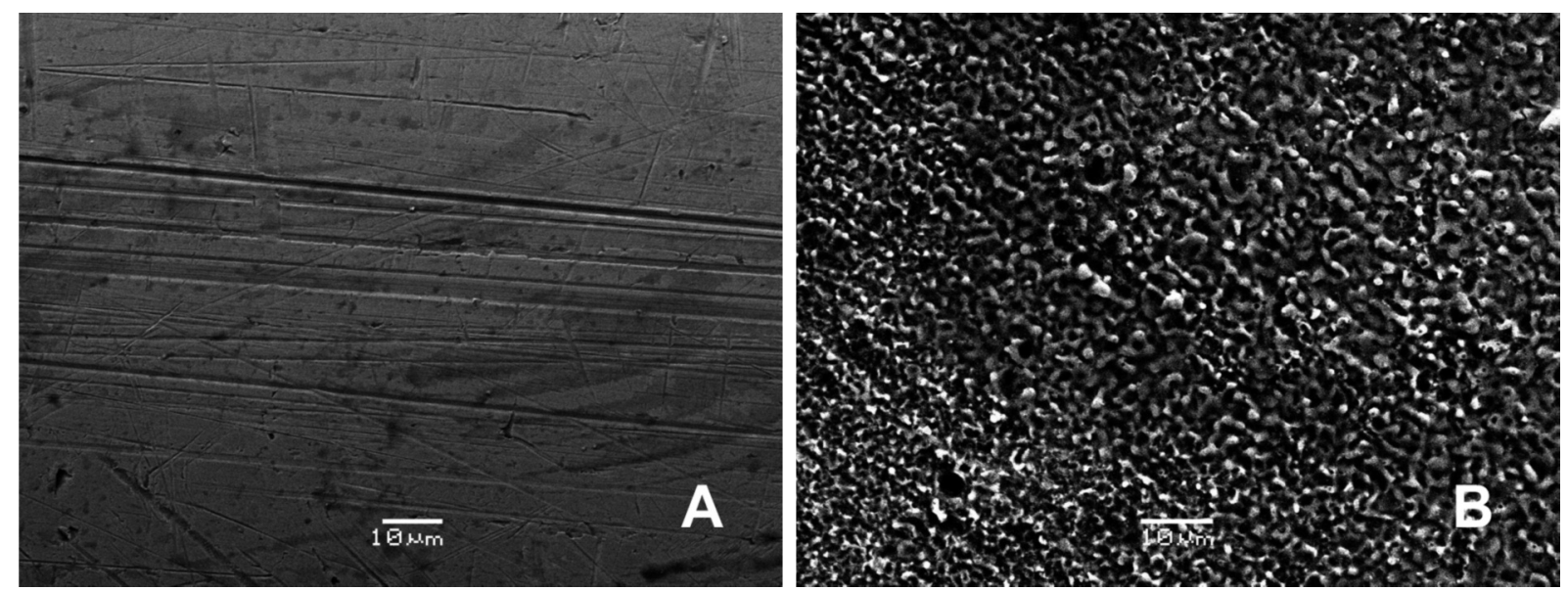

Figura 2. Superficie de acero inoxidable 302 comercial (A) y tratado por PE durante $10 \mathrm{~s}$ (B).

Figure 2. Surface morphology of as received samples $(A)$ and treated with electrolytic plasma $(B)$. 
En ella se puede observar que el tratamiento por $\mathrm{PE}$ genera en la superficie la presencia uniforme de cráteres y nódulos propios de las descargas puntuales de plasma ${ }^{[16]}$. Esta superficie nos indica que la misma fue expuesta a sucesivos impactos térmicos de alcance menor a $5 \mu \mathrm{m}$, y temperatura mayor a la de fusión. Luego del ensayo de tracción, la superficie de las muestras A y B quedó de acuerdo a lo mostrado en la figura 3 adjunta, que muestra las microfotografías obtenidas en la zona inmediata a la fractura.

En la superficie de las muestras sometidas a PE (B) se observan los nódulos y cráteres alineados en el sentido de la tracción, pero sin fracturas ni separaciones, que corresponden a los defectos típicos de iniciación de la ruptura.

\subsection{Copas de ruptura}

La figura 4 muestra la copa de ruptura obtenida para las probetas A y B.

En la probeta comercial A se observa una ruptura dúctil, asociada a la formación de un profundo plano de desprendimiento a $40-45^{\circ}$ respecto de la dirección de la tracción.

Por otro lado, en la probeta B se observa estricción y un desprendimiento diagonal sólo en un anillo perimetral, perfectamente diferenciado de un plano de ruptura central. En particular, la figura 4 (B) permite cuantificar entre 12 y $20 \mu \mathrm{m}$ el ancho del citado anillo perimetral, sección en la que existe un hábito cris-
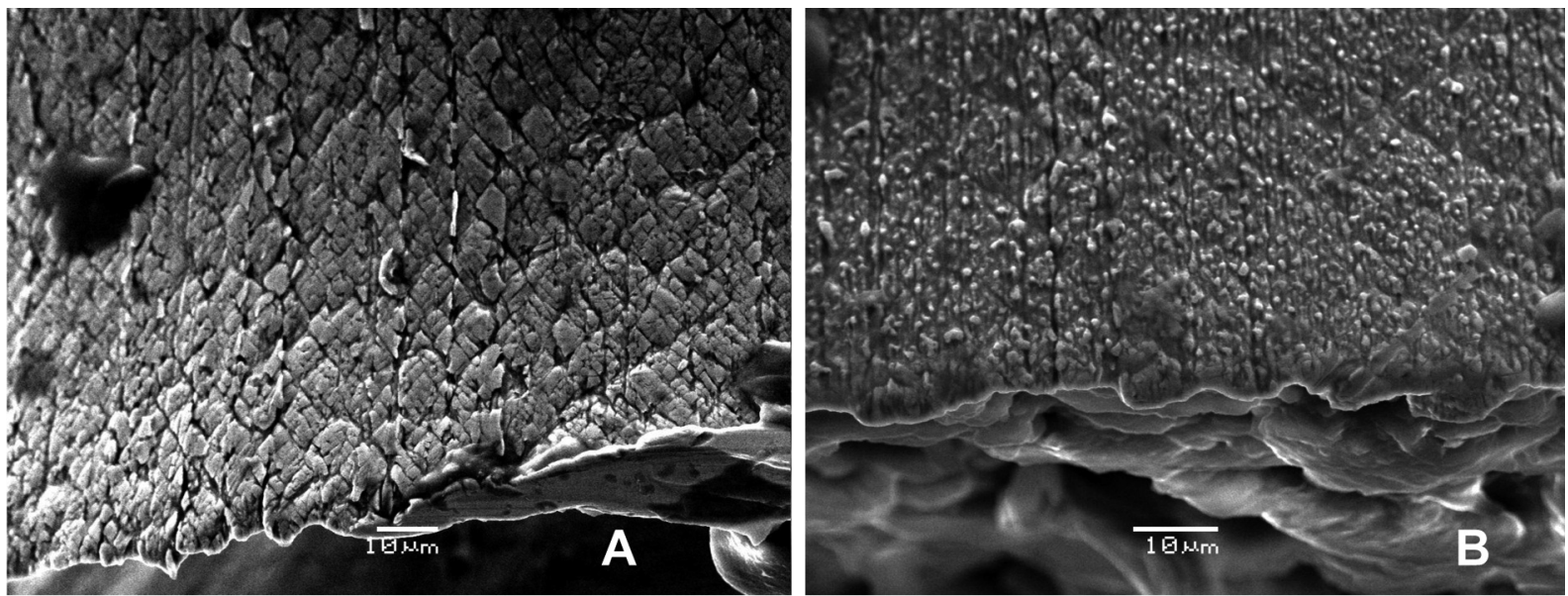

Figura 3. Superficie de las muestras comerciales (A) y con PE (B) en las inmediaciones de la ruptura.

Figure 3. Surface morphology of as received samples $(A)$ and treated with electrolytic plasma $(B)$ in the vicinity of the rupture.
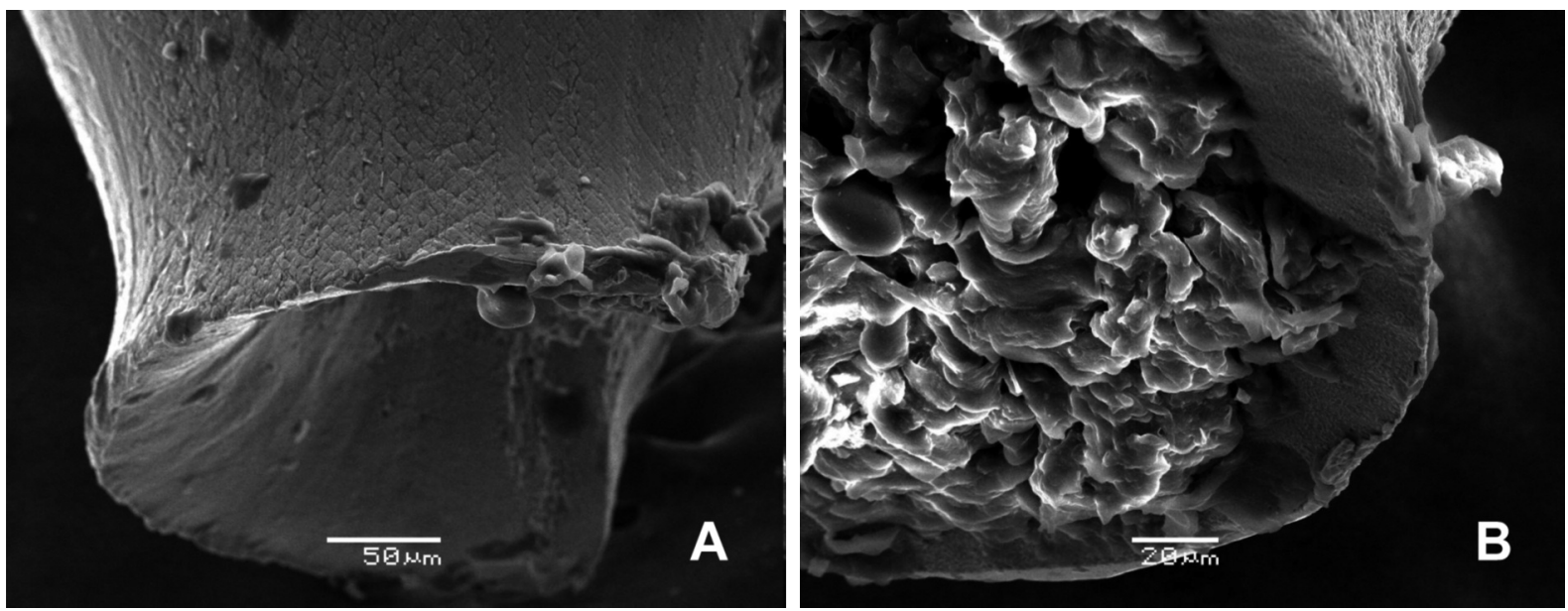

Figura 4. Copa de ruptura de las muestras comerciales $(A)$ y con $P E(B)$.

Figure 4. Separation plane of the samples as received $(A)$ and treated with electrolytic plasma $(B)$. 
talográfico muy fino formado por el PE. La zona central presenta una ruptura perpendicular respecto de la dirección de tracción, asociada a una fractura más bien de tipo frágil. La figura 4 (B) también permite observar que no existen fallas de adherencia entre la zona central y perimetral, lo que fue confirmado al observar la interfase en forma extensa mediante MEB a aumentos superiores.

\subsection{Análisis metalográfico}

Se prepararon secciones metalográficas de las muestras A y B, lo que resultó particularmente complejo, debido a la elevada resistencia al ataque químico presentada por la capa externa de las probetas sometidas a $P E^{[16]}$. La figura 5 muestra la sección de las probetas A y B, con magnificación de 500X.

En la muestra B se puede observar la aparición de rugosidad superficial y una zona perimetral de afinamiento de grano, progresiva hacia la superficie de la muestra, que posee una profundidad aproximada de $50 \mu \mathrm{m}$. En el borde de las muestras, los límites de grano tienden a desaparecer, observándose una capa de espesor variable, en la que no se observan granos. Estas últimas observaciones incentivaron el estudio del tamaño de los ordenamientos cristalinos superficiales que resultan en la muestra luego de someterla al tratamiento por PE.

\subsection{Análisis por STEM}

Se preparó una sección superficial con transparencia electrónica de la muestra B, que luego fue observada por STEM, obteniéndose la micrografía de la figura 6 .

Esta imagen suministra información de la capa más externa de la zona afectada por el $\mathrm{PE}$, en particular, de las primeras $2 \mu \mathrm{m}$. En ella se pueden observar granos alargados en el borde de la muestra, de $100 \mathrm{~nm}$ de sección por 300 a $400 \mathrm{~nm}$ de profundidad, seguidos de un lecho de más de $500 \mathrm{~nm}$ de espesor compuesto por granos de tamaño menor a $150 \mathrm{~nm}$. La presencia de granos alargados en el exterior de la muestra, se explica por la dirección del flujo térmico de enfriamiento, que es desde la muestra hacia el exterior, la zona de contacto con el electrolito.

\subsection{Ensayos de tracción}

Para evaluar el efecto del tratamiento por PE en la resistencia a la tracción, se determinó la carga máxima de las probetas A y B. En todos los ensayos las probetas presentaron una cierta estricción del alambre. Los valores de carga máxima que soportaron las probetas sometidas a PE mostraron un aumento de 8,8 y $9,8 \%$ para velocidades de cabezal de 0,0167 y $0,0017 \mathrm{~mm} / \mathrm{s}$ respectivamente, de acuerdo a la tabla II.

Sin embargo, si se compara la resistencia a la tracción de la capa con plasma con su equivalente sin tratamiento, los resultados de la primera son notablemente superiores, tal como se muestra a continuación.

Si se considera que existen sólo dos zonas en las probetas tratadas, la perimetral y el bulk, siendo este último un material que mantiene sus características
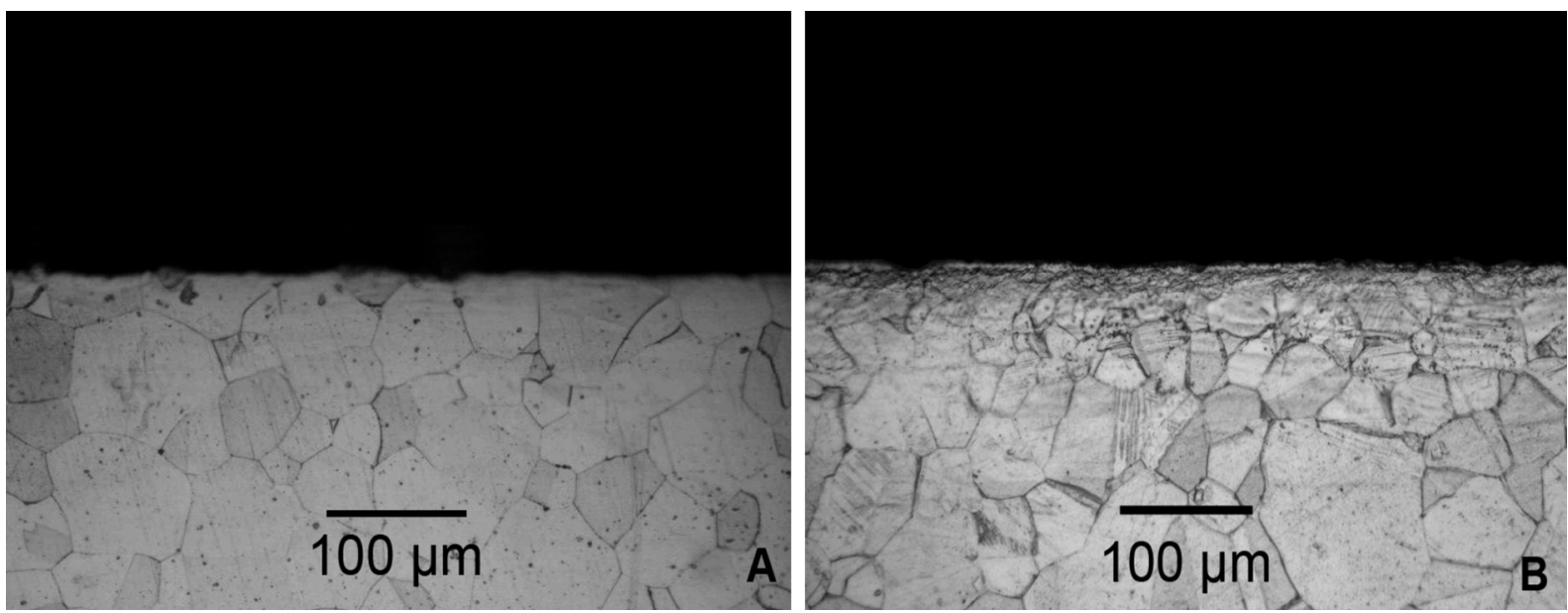

Figura 5. Metalografías de sección externa de probetas A y B, ambas con aumento de 500X.

Figure 5. Microstructures of external section of probes $A$ and $B$, both with 500X. 
Tabla II. Resultados de los ensayos de tracción

Table II. Results of tensile assays

\begin{tabular}{|c|c|c|c|c|}
\hline Ensayo & Condición & Velocidad & Carga máxima (kg) & Resistencia tracción \\
\hline \multirow[t]{2}{*}{$\begin{array}{l}1 \\
2 \\
3\end{array}$} & Comercial & $0,0167 \mathrm{~mm} / \mathrm{s}$ & $\begin{array}{l}39,15 \\
40,67 \\
38,50\end{array}$ & $\begin{array}{l}199,39 \\
207,13 \\
196,08\end{array}$ \\
\hline & \multicolumn{2}{|c|}{ Resistencia a la tracción $\left(\mathrm{kg} / \mathrm{mm}^{2}\right)$} & & 200,87 \\
\hline \multirow[t]{2}{*}{$\begin{array}{l}4 \\
5 \\
6\end{array}$} & PE $10 \mathrm{~s}$ & $0,0167 \mathrm{~mm} / \mathrm{s}$ & $\begin{array}{l}41,75 \\
43,73 \\
43,25\end{array}$ & $\begin{array}{l}212,63 \\
222,72 \\
220,27\end{array}$ \\
\hline & \multicolumn{2}{|c|}{ Resistencia a la tracción $\left(\mathrm{kg} / \mathrm{mm}^{2}\right)$} & & 218,54 \\
\hline \multirow[t]{2}{*}{$\begin{array}{l}7 \\
8 \\
9\end{array}$} & Comercial & $0,0017 \mathrm{~mm} / \mathrm{s}$ & $\begin{array}{l}38,70 \\
38,25 \\
38,40\end{array}$ & $\begin{array}{l}197,10 \\
194,81 \\
195,57\end{array}$ \\
\hline & \multicolumn{2}{|c|}{ Resistencia a la tracción $\left(\mathrm{kg} / \mathrm{mm}^{2}\right)$} & & 195,82 \\
\hline \multirow[t]{2}{*}{$\begin{array}{l}10 \\
11 \\
12\end{array}$} & PE $10 \mathrm{~s}$ & $0,0017 \mathrm{~mm} / \mathrm{s}$ & $\begin{array}{l}43,01 \\
42,12 \\
41,70\end{array}$ & $\begin{array}{l}219,05 \\
214,52 \\
212,38\end{array}$ \\
\hline & \multicolumn{2}{|c|}{ Resistencia a la tracción $\left(\mathrm{kg} / \mathrm{mm}^{2}\right)$} & & 215,31 \\
\hline
\end{tabular}

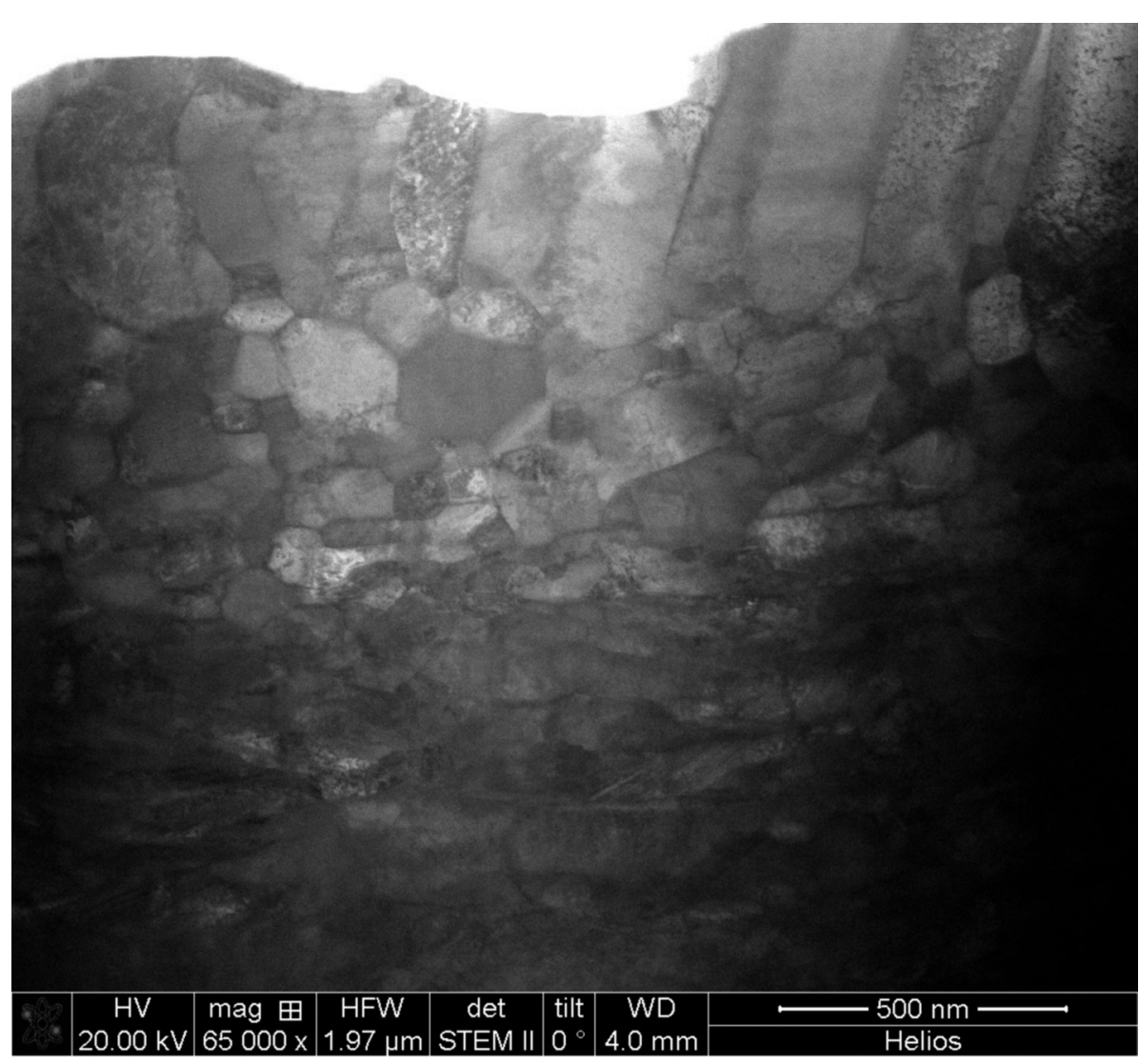

Figura 6. Micrografía STEM de granos en la zona perimetral de la muestra sometida a PE.

Figure 6. STEM micrograph of grains at the outer edges of the sample treated by EP. 
Tabla III. Resistencia a la tracción calculada para distintos espesores de capa externa

Table III. Tensile strength calculated for different thicknesses of outer layer

\begin{tabular}{lccc}
\hline $\begin{array}{c}\text { Espesor } \\
(\boldsymbol{\mu m})\end{array}$ & $\begin{array}{c}\boldsymbol{\sigma} \text { bulk } \\
\left(\mathbf{k g} / \mathbf{m m}^{2}\right)\end{array}$ & $\begin{array}{c}\boldsymbol{\sigma} \mathbf{p e} \\
\left(\mathbf{k g} / \mathbf{m m}^{\mathbf{2}}\right)\end{array}$ & $\begin{array}{c}\text { Incremento } \\
\mathbf{\%}\end{array}$ \\
\hline 12,5 & 200,9 & 382,1 & 90,2 \\
15 & 200,9 & 352,7 & 75,6 \\
17,5 & 200,9 & 331,7 & 65,1 \\
20 & 200,9 & 315,9 & 57,3 \\
12,5 & 195,8 & 395,7 & 102,1 \\
15 & 195,8 & 363,3 & 85,5 \\
17,5 & 195,8 & 340,1 & 73,7 \\
20 & 195,8 & 322,7 & 64,8 \\
\hline
\end{tabular}

originales, entonces la expresión para la fuerza máxima queda como sigue, (Ec. 1):

$$
F_{r}=\sigma_{\text {bulk }} * A_{\text {bulk }}+\sigma_{p e} * A_{p e}
$$

Donde:

$\mathrm{F}_{\mathrm{r}}$ : Fuerza máxima registrada en el ensayo de tracción $(\mathrm{kg})$.

$\sigma_{\text {bulk }}$ : Resistencia a la tracción registrada en las probetas sin tratamiento $\left(\mathrm{kg} / \mathrm{mm}^{2}\right)$.

$\sigma_{\mathrm{pe}}$ : Resistencia a la tracción de la zona afectada por ele PE.

el PE.

$\mathrm{A}_{\text {bulk }}$ : Área de la sección interna, no afectada por

$\mathrm{A}_{\mathrm{pe}}$ : Área de la sección perimetral afectada $\left(\mathrm{mm}^{2}\right)$.

Los resultados obtenidos, considerando espesores de capa tratada entre 12,5 y $20 \mu \mathrm{m}$, se muestran en la tabla III; en ella se puede apreciar que la zona tratada aumenta su resistencia a la tracción en al menos un $57 \%$ respecto del material original.

Cabe destacar que no se consideró en los cálculos la zona afectada térmicamente por el tratamiento de $\mathrm{PE}$, es decir, la comprendida entre los 20 y $50 \mu \mathrm{m}$, lo que permite suponer que los valores presentados son por defecto.

\section{CONCLUSIONES}

- La aplicación de PE durante 10 s permite formar una capa superficial de espesor variable, del orden de $15 \mu \mathrm{m}$, que otorga mejores características de mayor resistencia mecánica a las probetas. En particular, la capa más externa, de aproximadamente $500 \mathrm{~nm}$ de espesor, presenta ordenamien- tos cristalinos de tamaño nanoscópico, con excelentes propiedades de adherencia y estabilidad estructural, toda vez que no se observan fracturas ni desprendimientos en las zonas inmediatas a las copas de fractura.

- La capa superficial posee una resistencia a la tracción superior en al menos un 57 \% respecto de la condición original.

\section{Agradecimientos}

Los autores agradecen el apoyo de CONICYT Chile en la realización de este estudio. Además, las evaluaciones mediante STEM fueron realizadas en el marco del proyecto de cooperación NanoCom Network Project, 7th Frame- work program - IRSES - Project N 8247524.

\section{REFERENCIAS}

[1] A. Azushima, R. Kopp, A. Korhonen, D.Y. Yang, F. Micari, G.D. Lahoti, P. Groche, J. Yanagimoto, N. Tsuji, A. Rosochowski y A. Yanagida, Manufacturing Technology 57 (2008) 716-735.

[2] L. Peraldo-Bicelli, B. Bozzini, C. Mele y L. D'Urzo, Int. J. Electrochem. Sci. 3 (2008) 356-408.

[3] X. Sauvage, A. Chbihi y X. Quelennec, J. Phys. Conference Series 240 (2010) 012003.

[4] A. Rezaee, A. Najafizadeh, A. Kermanpur y M. Moallemi, Mater. Design. 32 (2011) 4.437-4.442.

[5] M. Multigner, E. Frutos, J. L. González-Carrasco, J. A. Jiménez, P. Marín y J. Ibáñez, Mater. Sci. Eng. C-Bio S, 29 (2009) 1.357-1.360.

[6] X.Y. Wang y D.Y. Li, Electrochim. Acta 47 (2002) 3.939-3.947.

[7] X.Y. Wang y D.Y. Li, Wear 255 (2003) 836-845.

[8] L. Wang y D.Y. Li, Surf. Coat. Tech. 167 (2003) 188-196.

[9] H. Yun-wei, D. Bo, Z. Cheng, J. Yi-rning y L. Jin, J. Iron Steel Res. Int. 16 (2009) 8-72.

[10] X.H. Chen, J. Lu, L. Lu y K. Lu, Scripta Mater. 52 (2005) 1.039-1.044.

[11] M. Ya, Y. Xing, F. Dai, K. Lu y J. Lu, Surf. Coat. Tech. 168 (2003) 148-155.

[12] C.T. Kwok, F.T. Cheng, H.C. Man y W.H. Ding, Mater. Lett. 60 (2006) 2.419-2.422.

[13] S. Barriuso, M. Lieblich, M. Multigner, I. Etxeberria, A. Alberdi y J.L. González-Carrasco, Wear 270 (2011) 634-639.

[14] D. Bedorf y S.G. Mayr, Scripta Mater. 57 (2007) 853-856. 
[15] C. Cui, J. Hu, Y. Liu, K. Gao y Z. Guo, Appl. Surf. Sci. 254 (2008) 6.779-6.782.

[16] P. Gupta, G. Tenhundfeld, E.O. Daigle y D. Ryabkov, Surf. Coat. Tech. 201 (2007) 8.7468.760 .

[17] H. H. Kellogg, J. Electrochem. Soc. 97 (1950) 133.

[18] A. Hickling y M. D. Ingram, Trans. Faraday Soc. 60 (1964) 783.

[19] D.I. Slovetskii y S. D. Terent`ev, High Energ. Chem., 37 (2003) 310.

[20] C. W. M. P. Sillen, E. Barendrecht, L .J. J. Janssen y S.J. D. van Stralen, Int. J. Hydrog. Energ. 7 (1982) 577.
[21] E.V. Parfenov, A. L.Yerokhin y A. Matthews, Surf. Coat. Tech. 201 (2007) 8.661-8.670.

[22] A.I. Maximov y A.V. Khlustova, Surf. Coat. Tech. 201 (2007) 8.782-8.788.

[23] E.I. Meletis, X. Nie, F.L. Wang y J.C. Jiang, Surf. Coat. Tech. 150 (2002) 246-256.

[24] T. Paulmier, J. M. Bell y P.M. Fredericks, Thin. Solid. Films. 515 (2007) 2.926-2.934.

[25] G. Sundararajan y L. Rama Krishna, Surf. Coat. Tech. 167 (2003) 269-277.

[26] J. Gao, A. Wang, Y. Li, Y. Fu, J. Wu, Youdi Wang y Yujing Wang, React. Funct. Polym. 68 (2008) 1.377-1.383.

[27] Q. Lu, J. Yu y J. Gao, J. Hazard Mater. B136 (2006) 526-531. 